\title{
Variations on Safety
}

\author{
Orna Kupferman
}

Hebrew University, School of Engineering and Computer Science, Jerusalem 91904, Israel orna@cs.huji.ac.il

\begin{abstract}
Of special interest in formal verification are safety properties, which assert that the system always stays within some allowed region, in which nothing "bad" happens. Equivalently, a property is a safety property if every violation of it occurs after a finite execution of the system. Thus, a computation violates the property if it has a "bad prefix", all whose extensions violate the property. The theoretical properties of safety properties as well as their practical advantages with respect to general properties have been widely studied. The paper surveys several extensions and variations of safety. We start with bounded and checkable properties - fragments of safety properties that enable an even simpler reasoning. We proceed to a reactive setting, where safety properties require the system to stay in a region of states that is both allowed and from which the environment cannot force it out. Finally, we describe a probability-based approach for defining different levels of safety.
\end{abstract}

\section{Introduction}

Today's rapid development of complex and safety-critical systems requires reliable verification methods. In formal verification, we verify that a system meets a desired property by checking that a mathematical model of the system meets a formal specification that describes the property. Of special interest are properties asserting that the observed behavior of the system always stays within some allowed region, in which nothing "bad" happens. For example, we may want to assert that every message sent is acknowledged in the next cycle. Such properties of systems are called safety properties. Intuitively, a property $\psi$ is a safety property if every violation of $\psi$ occurs after a finite execution of the system. In our example, if in a computation of the system a message is sent without being acknowledged in the next cycle, this occurs after some finite execution of the system. Also, once this violation occurs, there is no way to "fix" the computation.

In order to formally define what safety properties are, we refer to computations of a nonterminating system as infinite words over an alphabet $\Sigma$. Consider a language $L$ of infinite words over $\Sigma$. A finite word $x$ over $\Sigma$ is a bad prefix for $L$ iff for all infinite words $y$ over $\Sigma$, the concatenation $x \cdot y$ of $x$ and $y$ is not in $L$. Thus, a bad prefix for $L$ is a finite word that cannot be extended to an infinite word in $L$. A language $L$ is a safety language if every word not in $L$ has a finite bad prefix. For example, if $\Sigma=\{0,1\}$, then $L=\left\{0^{\omega}, 1^{\omega}\right\}$ is a safety language. Indeed, every word not in $L$ contains either the sequence 01 or the sequence 10 , and a prefix that ends in one of these sequences cannot be extended to a word in $L$.1.

\footnotetext{
${ }^{1}$ The definition of safety we consider here is given in [1|2], it coincides with the definition of limit closure defined in [12], and is different from the definition in [26], which also refers to the property being closed under stuttering.
} 
The interest in safety started with the quest for natural classes of specifications. The theoretical aspects of safety have been extensively studied [2 28 29 33]. With the growing success and use of formal verification, safety has turned out to be interesting also from a practical point of view [14|20|23]. Indeed, the ability to reason about finite prefixes significantly simplifies both enumerative and symbolic algorithms. In the first, safety circumvents the need to reason about complex $\omega$-regular acceptance conditions. For example, methods for temporal synthesis, program repair, or parametric reasoning are much simpler for safety properties [18|32]. In the second, it circumvents the need to reason about cycles, which is significant in both BDD-based and SAT-based methods [5[6]. In addition to a rich literature on safety, researchers have studied additional classes, such as liveness and co-safety properties [2|28].

The paper surveys several extensions and variations of safety. We start with bounded and checkable properties - fragments of safety properties that enable an even simpler reasoning. We proceed to a reactive setting, where safety properties require the system to stay in a region of states that is both allowed and from which the environment cannot force it out. Finally, we describe a probability-based approach for defining different levels of safety. The survey is based on the papers [24], with Moshe Y. Vardi, [21], with Yoad Lustig and Moshe Y. Vardi, [25], with Sigal Weiner, and [10], with Shoham Ben-David.

\section{Preliminaries}

Safety and Co-Safety Languages. Given an alphabet $\Sigma$, a word over $\Sigma$ is a (possibly infinite) sequence $w=\sigma_{0} \cdot \sigma_{1} \cdots$ of letters in $\Sigma$. Consider a language $L \subseteq \Sigma^{\omega}$ of infinite words. A finite word $x \in \Sigma^{*}$ is a bad prefix for $L$ iff for all $y \in \Sigma^{\omega}$, we have $x \cdot y \notin L$. Thus, a bad prefix is a finite word that cannot be extended to an infinite word in $L$. Note that if $x$ is a bad prefix, then all the finite extensions of $x$ are also bad prefixes. A language $L$ is a safety language iff every infinite word $w \notin L$ has a finite bad prefix. For a safety language $L$, we denote by bad-pref $(L)$ the set of all bad prefixes for $L$.

For a language $L \subseteq \Sigma^{\omega}$, we use $\operatorname{comp}(L)$ to denote the complement of $L$; i.e., $\operatorname{comp}(L)=\Sigma^{\omega} \backslash L$. A language $L \subseteq \Sigma^{\omega}$ is a co-safety language iff $\operatorname{comp}(L)$ is a safety language. (The term used in [28] is guarantee language.) Equivalently, $L$ is co-safety iff every infinite word $w \in L$ has a good prefix $x \in \Sigma^{*}$ : for all $y \in \Sigma^{\omega}$, we have $x \cdot y \in L$. For a co-safety language $L$, we denote by good-pref $(L)$ the set of good prefixes for $L$. Note that for a safety language $L$, we have that good-pref $(\operatorname{comp}(L))=$ bad-pref $(L)$.

Word Automata. A nondeterministic Büchi word automaton (NBW, for short) is $\mathcal{A}=$ $\left\langle\Sigma, Q, \delta, Q_{0}, F\right\rangle$, where $\Sigma$ is the input alphabet, $Q$ is a finite set of states, $\delta: Q \times \Sigma \rightarrow$ $2^{Q}$ is a transition function, $Q_{0} \subseteq Q$ is a set of initial states, and $F \subseteq Q$ is a set of accepting states. If $\left|Q_{0}\right|=1$ and $\delta$ is such that for every $q \in Q$ and $\sigma \in \Sigma$, we have that $|\delta(q, \sigma)| \leq 1$, then $\mathcal{A}$ is a deterministic Büchi word automaton (DBW, for short).

Given an input word $w=\sigma_{0} \cdot \sigma_{1} \cdots$ in $\Sigma^{\omega}$, a run of $\mathcal{A}$ on $w$ is a sequence $r_{0}, r_{1}, \ldots$ of states in $Q$ such that $r_{0} \in Q_{0}$ and for every $i \geq 0$, we have $r_{i+1} \in \delta\left(r_{i}, \sigma_{i}\right)$. For a run $r$, let $\inf (r)$ denote the set of states that $r$ visits infinitely often. That is, 
$\inf (r)=\left\{q \in Q: r_{i}=q\right.$ for infinitely many $\left.i \geq 0\right\}$. As $Q$ is finite, it is guaranteed that $\inf (r) \neq \emptyset$. The run $r$ is accepting iff $\inf (r) \cap F \neq \emptyset$. That is, iff there exists a state in $F$ that $r$ visits infinitely often. A run that is not accepting is rejecting. When $\alpha=Q$, we say that $\mathcal{A}$ is a looping automaton. We use NLW and DLW to denote nondeterministic and deterministic lopping automata. An NBW $\mathcal{A}$ accepts an input word $w$ iff there exists an accepting run of $\mathcal{A}$ on $w$. The language of an NBW $\mathcal{A}$, denoted $\mathcal{L}(\mathcal{A})$, is the set of words that $\mathcal{A}$ accepts. We assume that a given NBW $\mathcal{A}$ has no empty states, except maybe the initial state (that is, at least one word is accepted from each state otherwise we can remove the state).

Linear Temporal Logic. The logic LTL is a linear temporal logic. Formulas of LTL are constructed from a set $A P$ of atomic propositions using the usual Boolean operators and the temporal operators $G$ ("always"), $F$ ("eventually"), $X$ ("next time"), and $U$ ("until"). Formulas of LTL describe computations of systems over $A P$. For example, the LTL formula $G(r e q \rightarrow F a c k)$ describes computations in which every position in which req holds is eventually followed by a position in which ack holds. Thus, each LTL formula $\psi$ corresponds to a language, denoted $\|\psi\|$, of words in $\left(2^{A P}\right)^{\omega}$ that satisfy it. For the detailed syntax and semantics of LTL, see [30]. The model-checking problem for LTL is to determine, given an LTL formula $\psi$ and a system $M$, whether all the computations of $M$ satisfy $\psi$.

General methods for LTL model checking are based on translation of LTL formulas to nondeterministic Büchi word automata. By [36], given an LTL formula $\psi$, one can construct an NBW $\mathcal{A}_{\psi}$ over the alphabet $2^{A P}$ that accepts exactly all the computations that satisfy $\psi$. The size of $\mathcal{A}_{\psi}$ is, in the worst case, exponential in the length of $\psi$.

Given a system $M$ and an LTL formula $\psi$, model checking of $M$ with respect to $\psi$ is reduced to checking the emptiness of the product of $M$ and $\mathcal{A}_{\neg \psi}$ [36]. This check can be performed on-the-fly and symbolically [735], and the complexity of model checking that follows is PSPACE, with a matching lower bound [34].

It is shown in [2 33|22] that when $\psi$ is a safety formula, we can assume that all the states in $\mathcal{A}_{\psi}$ are accepting. Indeed, $\mathcal{A}_{\psi}$ accepts exactly all words all of whose prefixes have at least one extension accepted by $\mathcal{A}_{\psi}$, which is what we get if we define all the states of $\mathcal{A}_{\psi}$ to be accepting. Thus, safety properties can be recognized by NLWs. Since every NLW can be determined to an equivalent DLW by applying the subset construction, all safety formulas can be translated to DLWs.

\section{Interesting Fragments}

In this section we discuss two interesting fragments of safety properties: clopen (a.k.a. bounded) properties, which are useful in bounded model checking, and checkable properties, which are useful in real-time monitoring.

\subsection{Clopen Properties}

Bounded model checking methodologies check the correctness of a system with respect to a given specification by examining computations of a bounded length. Results from 
set-theoretic topology imply that sets in $\Sigma^{\omega}$ that are both open and closed (clopen sets) are bounded: membership in a clopen set can be determined by examining a bounded number of letters in $\Sigma$.

In [24] we studied safety properties from a topological point of view. We showed that clopen sets correspond to properties that are both safety and co-safety, and show that when clopen specifications are given by automata or LTL formulas, we can point to a bound and translate the specification to bounded formalisms such as bounded LTL and cycle-free automata.

Topology. Consider a set $X$ and a distance function $d: X \times X \rightarrow \mathbb{R}$ between the elements of $X$. For an element $x \in X$ and $\gamma \geq 0$, let $K(x, \gamma)$ be the set of elements $x^{\prime}$ such that $d\left(x, x^{\prime}\right) \leq \gamma$. Consider a set $S \subseteq X$. An element $x \in S$ is called an interior element of $S$ if there is $\gamma>0$ such that $K(x, \gamma) \subseteq S$. The set $S$ is open if all the elements in $S$ are interior. A set $S$ is closed if $X \backslash S$ is open. So, a set $S$ is open if every element in $S$ has a nonempty "neighborhood" contained in $S$, and a set $S$ is closed if every element not in $S$ has a nonempty neighborhood whose intersection with $S$ is empty. A set that is both open and close is called a clopen set.

A Cantor space consists of $X=D^{\omega}$, for some finite set $D$, and $d$ defined by $d\left(w, w^{\prime}\right)=\frac{1}{2^{n}}$, where $n$ is the first position where $w$ and $w^{\prime}$ differ. Thus, elements of $X$ can be viewed as infinite words over $D$ and two words are close to each other if they have a long common prefix. If $w=w^{\prime}$, then $d\left(w, w^{\prime}\right)=0$. It is known that clopen sets in Cantor space are bounded, where a set $S$ is bounded if it is of the form $W \cdot D^{\omega}$ for some finite set $W \subseteq D^{*}$. Hence, clopen sets in our Cantor space correspond exactly to bounded properties: each clopen language $L \subseteq \Sigma^{\omega}$ has a bound $k \geq 0$ such that membership in $L$ can be determined by the prefixes of length $k$ of words in $\Sigma^{\omega}$.

It is not hard to see that a language $L \subseteq \Sigma^{\omega}$ is co-safety iff $L$ is an open set in our Cantor space [2717]. To see this, consider a word $w$ in a co-safety language $L$, and let $x$ be a good prefix of $w$. All the words $w^{\prime}$ with $d\left(w, w^{\prime}\right) \leq \frac{1}{2^{|x|}}$ have $x$ as their prefix, so they all belong to $L$. For the second direction, consider a word $w$ in an open set $L$, and let $\gamma>0$ be such that $K(w, \gamma) \subseteq L$. The prefix of $w$ of length $\left\lfloor\log \frac{1}{\gamma}\right\rfloor$ is a good prefix for $L$. It follows that the clopen sets in $\Sigma^{\omega}$ are exactly these properties that are both safety and co-safety!

Bounding Clopen Properties. Our goal in this section is to identify a bound for a clopen property given by an automaton. Consider a clopen language $L \subseteq \Sigma^{\omega}$. For a finite word $x \in \Sigma^{*}$, we say that $x$ is undetermined with respect to $L$ if there are $y \in \Sigma^{\omega}$ and $z \in \Sigma^{\omega}$ such that $x \cdot y \in L$ and $x \cdot z \notin L$. As shown in [24], every word in $\Sigma^{\omega}$ has only finitely many prefixes that are undetermined with respect to $L$. It follows that $L$ is bounded: there are only finitely many words in $\Sigma^{*}$ that are undetermined with respect to $L$. For an integer $k$, we say that $L$ is bounded by $k$ if all the words $x \in \Sigma^{*}$ such that $|x| \geq k$ are determined with respect to $L$. Moreover, since $L$ is bounded, then a minimal DLW that recognizes $L$ must be cycle free. Indeed, otherwise we can pump a cycle to infinitely many undetermined prefixes. Let diameter $(L)$ be the diameter of the minimal DLW for $L$.

Lemma 1. A clopen $\omega$-regular language $L \subseteq \Sigma^{\omega}$ is bounded by diameter $(L)$. 
Proof: Let $\mathcal{A}$ be the minimal deterministic looping automaton for $L$. Consider a word $x \in \Sigma^{*}$ with $|x| \geq$ diameter $(L)$. Since $\mathcal{A}$ is cycle free, its run on $x$ either reaches an accepting sink, in which case $x$ is a good prefix, or it does not reach an accepting sink, in which case, by the definition of diameter $(\mathcal{A})$, we cannot extend $x$ to a word accepted by $\mathcal{A}$, thus $x$ is a bad prefix.

For a language $L$, the in index of $L$, denoted inindex $(L)$, is the minimal number of states that an NBW recognizing $L$ has. Similarly, the out index of $L$, denoted outindex $(L)$, is the minimal number of states that an NBW recognizing $\operatorname{comp}(L)$ has.

Lemma 2. A clopen $\omega$-regular language $L \subseteq \Sigma^{\omega}$ is bounded by inindex $(L)$. outindex $(L)$.

Proof: Assume by way of contradiction that there is a word $x \in \Sigma^{*}$ such that $|x| \geq$ inindex $(L) \cdot$ outindex $(L)$ and $x$ is undetermined with respect to $L$. Thus, there are suffixes $y$ and $z$ such that $x \cdot y \in L$ and $x \cdot z \notin L$. Let $\mathcal{A}_{1}$ and $\mathcal{A}_{2}$ be nondeterministic looping automata such that $\mathcal{L}\left(\mathcal{A}_{1}\right)=L, \mathcal{L}\left(\mathcal{A}_{2}\right)=\operatorname{comp}(L)$, and $\mathcal{A}_{1}$ and $\mathcal{A}_{2}$ have inindex $(L)$ and outindex $(L)$ states, respectively. Consider two accepting runs $r_{1}$ and $r_{2}$ of $\mathcal{A}_{1}$ and $\mathcal{A}_{2}$ on $x \cdot y$ and $x \cdot z$, respectively. Since $|x| \geq \operatorname{inindex}(L) \cdot \operatorname{outindex}(L)$, there are two prefixes $x[1, \ldots, i]$ and $x[1, \ldots, j]$ of $x$ such that $i<j$ and both runs repeat their state after these two prefixes; i.e., $r_{1}(i)=r_{1}(j)$ and $r_{2}(i)=r_{2}(j)$. Consider the word $x^{\prime}=x[1, \ldots, i] \cdot x[i+1, \ldots, j]^{\omega}$. Since $\mathcal{A}_{1}$ is a looping automaton, the run $r_{1}$ induces an accepting run $r_{1}^{\prime}$ of $\mathcal{A}_{1}$ on $x^{\prime}$. Formally, for all $l \leq i$ we have $r_{1}^{\prime}(l)=r_{1}(l)$ and for all $l>i$, we have $r_{1}^{\prime}(l)=r_{1}(i+((l-i) \bmod (j-i)))$. Similarly, the run $r_{2}$ induces an accepting run of $\mathcal{A}_{2}$ on $x^{\prime}$. It follows that $x^{\prime}$ is accepted by both $\mathcal{A}_{1}$ and $\mathcal{A}_{2}$, contradicting the fact that $\mathcal{L}\left(\mathcal{A}_{2}\right)=\operatorname{comp}\left(\mathcal{L}\left(\mathcal{A}_{1}\right)\right)$.

\subsection{Checkable Properties}

For an integer $k \geq 1$, a language $L \subseteq \Sigma^{\omega}$ is $k$-checkable if there is a language $R \subseteq$ $\Sigma^{k}$ (of "allowed subwords") such that a word $w$ belongs to $L$ iff all the subwords of $w$ of length $k$ belong to $R$. A property is locally checkable if its language is $k$ checkable for some $k$. Locally checkable properties, which are a special case of safety properties, are common in the specification of systems. In particular, one can often bound an eventuality constraint in a property by a fixed time frame, which results in a checkable property.

The practical importance of locally checkable properties lies in the low memory demand for their run-time verification. Indeed, $k$-checkable properties can be verified with a bounded memory - one that has access only to the last $k$-computation cycles. Run-time verification of a property amounts to executing a monitor together with the system allowing the detection of errors in run time [20339]. Run-time monitors for checkable specifications have low memory demand. Furthermore, in the case of general $\omega$-regular properties, when several properties are checked, we need a monitor for each property, and since the properties are independent of each other, so are the state spaces of the monitors. Thus, the memory demand (as well as the resources needed to maintain the memory) grow linearly with the number of properties monitored. Such a memory 
demand is a real problem in practice. In contrast, as shown in [21], a monitor for a $k$ checkable property needs only a record of the last $k$ computation cycles. Furthermore, even if a large number of $k$-checkable properties are monitored, the monitors can share their memory, resulting in memory demand of $|\Sigma|^{k}$, which is independant of the number of properties monitored.

As in the case of clopen properties, our goal is to identify a bound for a checkable property given by an automaton. We first need some notations. For a word $w \in \Sigma^{\omega}$ and $k \geq 0$, we denote by $\operatorname{sub}(w, k)$ the set of finite subwords of $w$ of length $k$, formally, $\operatorname{sub}(w, k)=\left\{y \in \Sigma^{*}:|y|=k\right.$ and there exist $x \in \Sigma^{*}$ and $z \in \Sigma^{\omega}$ such that $w=$ $x y z\}$. A language $L \subseteq \Sigma^{\omega}$ is $k$-checkable if there exists a finite language $R \subseteq \Sigma^{k}$ such that $w \in L$ iff all the $k$-long subwords of $w$ are in $R$. That is, $L=\{w \in$ $\left.\Sigma^{\omega}: \operatorname{sub}(w, k) \subseteq R\right\}$. A language $L \subseteq \Sigma^{\omega}$ is $k$-co-checkable if there exists a finite language $R \subseteq \Sigma^{k}$ such that $w \in L$ iff there exists a $k$-long subword of $w$ that is in $R$. That is, $L=\left\{w \in \Sigma^{\omega}: \operatorname{sub}(w, k) \cap R \neq \emptyset\right\}$. A language is checkable (cocheckable) if it is $k$-checkable ( $k$-co-checkable, respectively) for some $k$. We refer to $k$ as the width of $L$. It is easy to to see that all checkable languages are safety, and similarly for co-checkable and co-safety. In particular, $L$ is a checkable language induced by $R$ iff $\operatorname{comp}(L)$ is co-checkable and induced by $\operatorname{comp}(L)$.

In order to demonstrate the the subtlety of the width question, consider the following example.

Example 1. Let $\Sigma=\{0,1,2\}$. The DBW $\mathcal{A}$ below recognizes the language $L$ of all the words that contain 10,120 or 220 as subwords. Note that $L$ is the 3 -co-checkable language $L$ co-induced by $R=\{010,110,210,100,101,102,120,220\}$. Indeed, a word $w$ is in $L$ iff $\operatorname{sub}(w, 3) \cap R \neq \emptyset$.

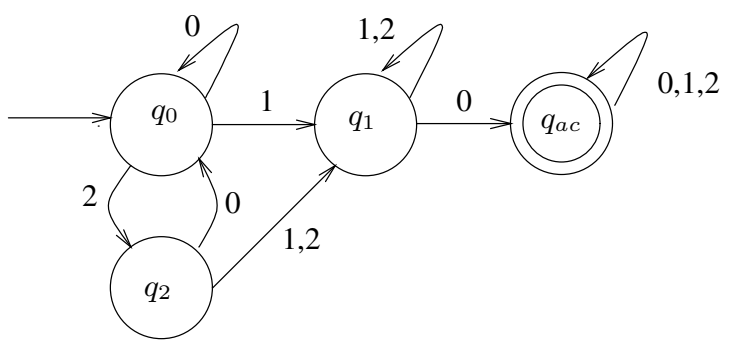

At first sight, it seems that the same considerations applied in Lemma 1 can be used in order to prove that the width of a checkable language is bounded by the diameter of the smallest DBW recognizing the language. Indeed, it appears that in an accepting run, the traversal through the minimal good prefix should not contain a cycle. This impression, however, is misleading, as demonstrated in the DBW $\mathcal{A}$ from Example 1 , where a traversal through the subword 120 contains a cycle, and similarly for 010 . The diameter of the DBW $\mathcal{A}$ is 3 , so it does not constitute a counterexample to the conjecture that the diameter bounds the width, but the problem remains open in [21], and the tightest bound proven there depends on the size of $\mathcal{A}$ and not only on its diameter, and is even not linear. Intuitively, it follows form an upper-bound on the size of a DBW that recognizes minimal bad prefixes of $L$. Formally, we have the following. 
Theorem 1. If a checkable (or co-checkable) language $L$ is recognized by a DBW with $n$ states, then the width of $L$ is bounded by $O\left(n^{2}\right)$.

As noted above, the bound in Theorem 1 is not tight and the best known lower bound is only the diameter of a DBW for $L$. For the nondeterministic setting the bound is tighter:

Theorem 2. If a checkable language $L$ is recognized by an NBW with $n$ states, then the width of $L$ is bounded by $2^{O(n)}$. Also, There exist an $N B W \mathcal{A}$ with $O(n)$ states such that $L(\mathcal{A})$ is $k$-checkable but not $(k-1)$-checkable, for $k=(n+1) 2^{n}+2$.

\section{Safety in a Reactive Setting}

Recall that safety is defined with respect to languages over an alphabet $\Sigma$. Typically, $\Sigma=2^{A P}$, where $A P$ is the set of the system's atomic propositions. Thus, the definition and studies of safety treat all the atomic propositions as equal and do not distinguish between input and output signals. As such, they are suited for closed systems - ones that do not maintain an interaction with their environment. In open (also called reactive) systems [1931], the system interacts with the environment, and a correct system should satisfy the specification with respect to all environments. A good way to think about the open setting is to consider the situation as a game between the system and the environment. The interaction between the players in this game generates a computation, and the goal of the system is that only computations that satisfy the specification will be generated.

Technically, one has to partition the set $A P$ of atomic propositions to a set $I$ of input signals, which the environment controls, and a set $O$ of output signals, which the system controls. An open system is then an I/O-transducer - a deterministic automaton over the alphabet $2^{I}$ in which each state is labeled by an output in $2^{O}$. Given a sequence of assignments to the input signals (each assignment is a letter in $2^{I}$ ), the run of the transducer on it induces a sequence of assignments to the output signals (that is, letters in $2^{O}$ ). Together these sequences form a computation, and the transducer realizes a specification $\psi$ if all its computations satisfy $\psi[31]$.

The transition from the closed to the open setting modifies the questions we typically ask about systems. Most notably, the synthesis challenge, of generating a system that satisfies the specification, corresponds to the satisfiability problem in the closed setting and to the realizability problem in the open setting. As another example, the equivalence problem between LTL specifications is different in the closed and open settings [16]. That is, two specifications may not be equivalent when compared with respect to arbitrary systems on $I \cup O$, but be open equivalent; that is, equivalent when compared with respect to $I / O$-transducers. To see this, note for example that a satisfiable yet non-realizable specification is equivalent to false in the open but not in the closed setting.

As mentioned above, the classical definition of safety does not distinguish between input and output signals. The definition can still be applied to open systems, as a special case of closed systems with $\Sigma=2^{I \cup O}$. In [11], Ehlers and Finkbeiner introduced reactive safety - a definition of safety for the setting of open systems. Essentially, reactive 
safety properties require the system to stay in a region of states that is both allowed and from which the environment cannot force it out. The definition in [11] is by means of sets of trees with directions in $2^{I}$ and labels in $2^{O}$. The use of trees naturally locate reactive safety between linear and branching safety. In [25], we suggested an equivalent yet differently presented definition, which explicitly use realizability, and study the theoretical aspects of receive safety and other reactive fragments of specifications. In this section, we review the definition and results from [25].

\subsection{Definitions}

We model open systems by transducers. Let $I$ and $O$ be finite sets of input and output signals, respectively. Given $x=i_{0} \cdot i_{1} \cdot i_{2} \cdots \in\left(2^{I}\right)^{\omega}$ and $y=o_{0} \cdot o_{1} \cdot o_{2} \cdots \in\left(2^{O}\right)^{\omega}$, we denote their composition by $x \oplus y=\left(i_{0}, o_{0}\right) \cdot\left(i_{1}, o_{1}\right) \cdot\left(i_{2}, o_{2}\right) \cdots \in\left(2^{I \cup O}\right)^{\omega}$. An $I / O$-transducer is a tuple $\mathcal{T}=\left\langle I, O, S, s_{0}, \eta, L\right\rangle$, where $S$ is a set of states, $s_{0} \in S$ is an initial state, $\eta: S \times 2^{I} \rightarrow S$ is a transition function, and $L: S \rightarrow 2^{O}$ is a labeling function. The run of $\mathcal{T}$ on a (finite or infinite) input sequence $x=i_{0} \cdot i_{1} \cdot i_{2} \cdots$, with $i_{j} \in 2^{I}$, is the sequence $s_{0}, s_{1}, s_{2}, \ldots$ of states such that $s_{j+1}=\eta\left(s_{j}, i_{j+1}\right)$ for all $j \geq 0$. The computation of $\mathcal{T}$ on $x$ is then $x \oplus y$, for $y=L\left(s_{0}\right) \cdot L\left(s_{1}\right) \cdot L\left(s_{2}\right) \cdots$ Note that $\mathcal{T}$ is responsive and deterministic (that is, it suggests exactly one successor state for each input letter), and thus $\mathcal{T}$ has a single run, generating a single computation, on each input sequence. We extend $\eta$ to finite words over $2^{I}$ in the expected way. In particular, $\eta\left(s_{0}, x\right)$, for $x \in\left(2^{I}\right)^{*}$ is the $|x|$-th state in the run on $x$. A transducer $\mathcal{T}$ induces a strategy $f:\left(2^{I}\right)^{*} \rightarrow 2^{O}$ such that for all $x \in\left(2^{I}\right)^{*}$, we have that $f(x)=L\left(\eta\left(s_{0}, x\right)\right)$. Given an LTL formula $\psi$ over $I \cup O$, we say that $\psi$ is $I / O$-realizable if there is a finitestate $I / O$-transducer $\mathcal{T}$ such that all the computations of $\mathcal{T}$ satisfy $\psi[31]$. We then say that $\mathcal{T}$ realizes $\psi$. When it is clear from the context, we refer to $I / O$-realizability as realizability, or talk about realizability of languages over the alphabet $2^{I \cup O}$.

Since the realizability problem corresponds to deciding a game between the system and the environment, and the game is determined [15], realizability is determined too, in the sense that either there is an $I / O$-transducer that realizes $\psi$ (that is, the system wins) or there is an $O / I$-transducer that realizes $\neg \psi$ (that is, the environment wins). Note that in an $O / I$-transducer the system and the environment "switch roles" and the system is the one that provides the inputs to the transducer. A technical detail is that in order for the setting of $O / I$-realizability to be dual to the one in $I / O$-realizability we need, in addition to switching the roles and negating the specification, to switch the player that moves first and consider transducers in which the environment initiates the interaction and moves first. Since we are not going to delve into constructions, we ignore this point, which is easy to handle.

Let $I$ and $O$ be sets of input and output signals, respectively. Consider a language $L \subseteq\left(2^{I \cup O}\right)^{\omega}$. For a finite word $u \in\left(2^{I \cup O}\right)^{*}$, let $L^{u}=\{s: u \cdot s \in L\}$ be the set of all infinite words $s$ such that $u \cdot s \in L$. Thus, if $L$ describes a set of allowed computations, then $L^{u}$ describes the set of allowed suffixes of computations starting with $u$.

We say that a finite word $u \in\left(2^{I \cup O}\right)^{*}$ is a system bad prefix for $L$ iff $L^{u}$ is not realizable. Thus, a system bad prefix is a finite word $u$ such that after traversing $u$, the system does not have a strategy to ensure that the interaction with the environment would generate a computation in $L$. We use $\operatorname{sbp}(L)$ to denote the set of system bad 
prefixes for $L$. Note that by determinacy of games, whenever $L^{u}$ is not realizable by the system, then its complement is realizable by the environment. Thus, once a bad prefix has been generated, the environment has a strategy to ensure that the entire generated behavior is not in $L$.

A language $L \subseteq\left(2^{I \cup O}\right)^{\omega}$ is a reactive safety language if every word not in $L$ has a system bad prefix. Below are two examples, demonstrating that a reactive safety language need not be safe. Note that the other direction does hold: Let $L$ be a safe language. Consider a word $w \notin L$ and a bad prefix $u \in\left(2^{I \cup O}\right)^{*}$ of $w$. Since $u$ is a bad prefix, the set $L^{u}$ is empty, and is therefore unrealizable, so $u$ is also a system bad prefix. Thus, every word not in $L$ has a system bad prefix, implying that $L$ is reactively safe.

Example 2. Let $I=\{f i x\}, O=\{e r r\}, \psi=G(e r r \rightarrow F f i x)$, and $L=\|\psi\|$. Note that $\psi$ is realizable using the system strategy "never err". Also, $L$ is clearly not safe, as every prefix can be extended to one that satisfies $\psi$. On the other hand, $L$ is reactively safe. Indeed, every word not in $L$ must have a prefix $u$ that ends with $\{e r r\}$. Since $L^{u}=\|F f i x\|$, which is not realizable, we have that $u$ is a system bad prefix and $L$ is reactively safe.

Example 3. Let $I=\{f i x\}, O=\{e r r\}, \psi=G \neg$ err $\vee F G f i x$, and $L=\|\psi\|$. Note that $\psi$ is realizable using the system strategy "never err". Also, $L$ is clearly not safe. We show $L$ is reactively safe. Consider a word $w \notin L$. Since $w$ does not satisfy $G \neg e r r$, there must be a prefix $u$ of $w$ such that $u$ contains a position satisfying err. Since words with prefix $u$ do not satisfy $G \neg e r r$, we have that $L^{u}=\| F G$ fix $\|$, which is not realizable. Thus, $u$ is a system bad prefix and $L$ is reactively safe.

\subsection{Properties of Reactive Safety}

In the closed settings, the set bad-pref $(L)$ is closed under finite extensions for all languages $L \subseteq \Sigma^{\omega}$. That is, for every finite word $u \in$ bad-pref $(L)$ and finite extension $v \in \Sigma^{*}$, we have that $u \cdot v \in \operatorname{bad}$-pref $(L)$. This is not the case in the reactive setting:

Theorem 3. System bad prefixes are not closed under finite extension.

Proof: Let $I=\{f i x\}, O=\{e r r\}$, and $\psi=G(e r r \rightarrow X f i x) \wedge F G \neg e r r$. Thus, $\psi$ states that every error the system makes is fixed by the environment in the following step, and that there is a finite number of errors. Let $L=\|\psi\|$. Clearly, $\psi$ is realizable, as the strategy "never err" is a winning strategy for the system. Also, $L$ is reactively safe, as a word $w \notin L$ must have a prefix $u$ that ends in a position satisfying $e r r$, and $u$ is a system bad prefix. We show that $\operatorname{sbp}(L)$ is not closed under finite extensions. To see this, consider the word $w=(\{e r r, f i x\} \cdot\{f i x\})^{\omega}$. That is, the system makes an error on every odd position, and the environment always fixes errors. Since there are infinitely many errors in $w$, it does not satisfy $\psi$. The prefix $u=\{e r r, f i x\}$ of $w$ is a system bad prefix. Indeed, an environment strategy that starts with $\neg f i x$ is a winning strategy. On the other hand, $u$ 's extension $v=\{e r r, f i x\} \cdot\{f i x\}$ is not a system bad prefix. Indeed, $L^{v}$ is realizable using the winning system strategy "never err". 
Recall that reasoning about safety properties is easier than reasoning about general properties. In particular, rather than working with automata on infinite words, one can model check safety properties using automata (on finite words) for bad prefixes. The question is whether and how we can take advantage of reactive safety when the specification is not safe (but is reactively safe). In [11], the authors answered this question to the positive and described a transition from reactively safe to safe formulas. The translation is by means of nodes in the tree in which a violation starts. The translation from [25] we are going to describe here uses realizability explicitly, which we find simpler.

For a language $L \subseteq\left(2^{I \cup O}\right)^{\omega}$, we define $\operatorname{close}(L)=L \cap\{w: w$ has no system bad prefix for $L\}$. Equivalently, close $(L)=L \backslash\{w: w$ has a system bad prefix for $L\}$. Intuitively, we obtain $\operatorname{close}(L)$ by defining all the finite extensions of $\operatorname{sbp}(L)$ as bad prefixes. It is thus easy to see that $\operatorname{sbp}(L) \subseteq$ bad-pref $(\operatorname{close}(L))$.

As an example, consider again the specification $\psi=G(\mathrm{err} \rightarrow X \mathrm{fix}) \wedge F G \neg \mathrm{err}$, with $I=\{f i x\}, O=\{e r r\}$. An infinite word contains a system bad prefix for $\psi$ iff it has a position that satisfies $\mathrm{err}$. Accordingly, $\operatorname{close}(\psi)=G \neg$ err. As another example, let us add to $O$ the signal $a c k$, and let $\psi=G(e r r \rightarrow X(f i x \wedge F a c k))$, with $I=\{f i x\}$, $O=\{e r r, a c k\}$. Again, $\psi$ is reactively safe and an infinite word contains a system bad prefix for $\psi$ iff it has a position that satisfies err. Accordingly, close $(\psi)=G \neg$ err.

Our definition of close $(L)$ is sound, in the following sense:

Theorem 4. A language $L \subseteq\left(2^{I \cup O}\right)^{\omega}$ is reactively safe iff close $(L)$ is safe.

While $L$ and close $(L)$ are not equivalent, they are open equivalent [16]. Formally, we have the following.

Theorem 5. For every language $L \subseteq\left(2^{I \cup O}\right)^{\omega}$ and I/O-transducer $\mathcal{T}$, we have that $\mathcal{T}$ realizes $L$ iff $\mathcal{T}$ realizes close $(L)$.

It is shown in [11] that given an LTL formula $\psi$, it is possible to construct a deterministic looping word automaton for $\operatorname{close}(\psi)$ with doubly-exponential number of states. In fact, as suggested in [23], it is then possible to generate also a deterministic automaton for the bad prefixes of $\operatorname{close}(\psi)$. Note that when $L$ is not realizable, we have that $\epsilon \in \operatorname{sbp}(L)$, implying that $\operatorname{close}(L)=\emptyset$. It follows that we cannot expect to construct small automata for close $(L)$, even nondeterministic ones, as the realizability problem for LTL can be reduced to easy questions about them.

Theorem 5 implies that a reactive safety language $L$ is open equivalent to a safe language, namely close $(L)$. Conversely, open equivalence to a safe language implies reactive safety. This follows from the fact that if $L$ and $L^{\prime}$ are open-equivalent languages, then a prefix $x$ is a minimal system bad prefix in $L$ iff $x$ is a minimal system bad prefix in $L^{\prime}$. We can thus conclude with the following.

Theorem 6. A language $L$ is reactively safe iff $L$ is open equivalent to a safe language.

In the setting of open systems, dualization of specifications is more involved, as one has not only to complement the language but to also dualizes the roles of the system and the environment. Accordingly, we actually have four fragments of languages that are induced by dualization of the reactive safety definition. We define them by means of bad and good prefixes.

Consider a language $L \subseteq\left(2^{I \cup O}\right)^{\omega}$ and a prefix $u \in\left(2^{I \cup O}\right)^{*}$. We say that: 
- $u$ is a system bad prefix if $L^{u}$ is not $I / O$-realizable.

- $u$ is a system good prefix if $L^{u}$ is $I / O$-realizable.

- $u$ is an environment bad prefix if $L^{u}$ is not $O / I$-realizable.

- $u$ is an environment good prefix if $L^{u}$ is $O / I$-realizable.

Now, a language $L \subseteq\left(2^{I \cup O}\right)^{\omega}$ is a system (environment) safety language if every word not in $L$ has a system (environment, respectively) bad prefix. The language $L$ is a system (environment) co-safety language if every word in $L$ has a system (environment, respectively) good prefix. System safety and environment co-safety dualize each other: For every language $L \subseteq\left(2^{I \cup O}\right)^{\omega}$, we have that $L$ is system safe iff $\operatorname{comp}(L)$ is environment co-safe.

Since each language $L^{u}$ is either $I / O$-realizable or not $I / O$-realizable, and the same for $O / I$-realizability, all finite words are determined, in the following sense.

Theorem 7. Consider a language $L \subseteq\left(2^{I \cup O}\right)^{\omega}$. All finite words in $\left(2^{I \cup O}\right)^{*}$ are determined with respect to $L$. That is, every prefix is either system good or system bad, and either environment good or environment bad, with respect to $L$.

Note that while every prefix is determined, a word may have both system bad and system good prefixes, and similarly for the environment, which is not the case in the setting of closed systems. For example, recall the language $L=\| G(\operatorname{err} \rightarrow X f i x) \wedge$ $F G \neg e r r \|$, for $I=\{f i x\}$ and $O=\{e r r\}$. As noted above, the word ( $\{e r r, f i x\}$. $\{f i x\})^{\omega}$ has both a system bad prefix $\{e r r, f i x\}$, and a system good prefix $\{e r r, f i x\}$. $\{f i x\}$.

In Section 3.1 we showed that in the closed setting, the intersection of safe and cosafe properties induces the fragment of bounded properties. It is shown in [25] that boundedness in the open setting is more involved, as a computation may have both infinitely many good and infinitely many bad prefixes. It is still possible, however, to define reactive bounded properties and use their appealing practical advantages.

\section{A Spectrum between Safety and Co-safety}

Safety is a binary notion. A property may or may not satisfy the definition of safety. In this section we describe a probability-based approach for defining different levels of safety. The origin of the definition is a study of vacuity in model checking [423]. Vacuity detection is a method for finding errors in the model-checking process when the specification is found to hold in the model. Most vacuity algorithms are based on checking the effect of applying mutations on the specification. It has been recognized that vacuity results differ in their significance. While in many cases vacuity results are valued as highly informative, there are also cases in which the results are viewed as meaningless by users. In [10], we suggested a method for an automatic ranking of vacuity results according to their level of importance. Our method is based on the probability of the mutated specification to hold in a random computation. For example, two natural mutations of the specification $G(r e q \rightarrow$ Fready $)$ are $G(\neg r e q)$, obtained by mutating the subformula ready to false, and GFready, obtained by mutating the subformula $r e q$ to true. It is agreed that vacuity information about satisfying the first mutation is 
more alarming than information about satisfying the second. The framework in [10] formally explains this, as the probability of $G(\neg r e q)$ to hold in a random computation is 0 , whereas the probability of GFready is 1 . In this section we suggest to use probability also for defining levels of safety.

\subsection{The Probabilistic Setting}

Given a set $S$ of elements, a probability distribution on $S$ is a function $\mu: S \rightarrow[0,1]$ such that $\Sigma_{s \in S} \mu(s)=1$. Consider an alphabet $\Sigma$. A random word over $\Sigma$ is a word in which for all indices $i$, the $i$-th letter is drown uniformly at random. In particular, when $\Sigma=2^{A P}$, then a random computation $\pi$ is such that for each atomic proposition $q$ and for each position in $\pi$, the probability of $q$ to hold in the position is $\frac{1}{2}$. An equivalent definition of this probabilistic model is by means of the probabilistic labeled structure $\mathcal{U}_{\Sigma}$, which generates computations in a uniform distribution. Formally, $\mathcal{U}_{\Sigma}$ is a clique with $|\Sigma|$ states in which a state $\sigma \in \Sigma$ is labeled $\sigma$, is initial with probability $\frac{1}{|\Sigma|}$, and the probability to move from a state $\sigma$ to a state $\sigma^{\prime}$ is $\frac{1}{|\Sigma|}$.

We define the probability of a language $\mathcal{L} \subseteq \Sigma^{\omega}$, denoted $\operatorname{Pr}(\mathcal{L})$, as the probability of the event $\left\{\pi: \pi\right.$ is a path in $\mathcal{U}_{\Sigma}$ that is labeled by a word in $\left.\mathcal{L}\right\}$. Accordingly, for an LTL formula $\varphi$, we define $\operatorname{Pr}(\varphi)$ as the probability of the event $\{\pi: \pi$ is a path in $\mathcal{U}_{2 A P}$ that satisfies $\left.\varphi\right\}$. For example, the probabilities of $X p, G p$, and $F p$ are $\frac{1}{2}, 0$, and 1 , respectively. Using $\mathcal{U}_{\Sigma}$ we can reduce the problem of finding $\operatorname{Pr}(\varphi)$ to $\varphi$ 's model checking. Results on probabilistic LTL model checking [8] then imply that the problem of finding the probability of LTL formulas is PSPACE-complete.

First-order logic respects a 0/1-law: the probability of a formula to be satisfied in a random model is either 0 or 1 [13]. It is easy to see that a 0/1-law does not hold for LTL. For example, for an atomic proposition $p$, we have that $\operatorname{Pr}(p)=\frac{1}{2}$. Back to our safety story, it is not hard to see that $\operatorname{Pr}(G \xi)$, for a formula $\xi$ with $\operatorname{Pr}(\xi) \neq 1$, is 0 . Dually, $\operatorname{Pr}(F \xi)$, for a formula $\xi$ with $\operatorname{Pr}(\xi) \neq 0$ is 1 . Can we relate this to the fact that $G p$ is a safety property whereas $F p$ is a co-safety property? Or perhaps it has to do with $F p$ being a liveness property 2 This is not clear, as, for example, the probability of clopen formulas depends on finitely many events and can vary between 0 to 1 . As another example, consider the two possible semantics of the Until temporal operator. For the standard, strong, Until, which is not a safe, we have $\operatorname{Pr}(p U q)=\frac{2}{3}$. By changing the semantics of the Until to a weak one, we get the safety formula $p W q$, with $p W q=p U q \vee G p$. Still, $\operatorname{Pr}(p W q)=\operatorname{Pr}(p U q)$. Thus, the standard probabilistic setting does not suggest a clear relation between probability and different levels of safety.

We argue that we can still use the probabilistic approach in order to measure safety. The definition of $\operatorname{Pr}(\varphi)$ in [10] assumes that the probability of an atomic proposition to hold in each position is $\frac{1}{2}$. This corresponds to computations in an infinite-state system and is the standard approach taken in studies of 0/1-laws. Alternatively, one can also study the probability of formulas to hold in computations of finite-state systems. Formally, for an integer $l \geq 1$, let $\operatorname{Pr}_{l}(\varphi)$ denote the probability that $\varphi$ holds in a random cycle of length $l$. Here too, the probability of each atomic proposition to hold in a state is $\frac{1}{2}$, yet we have only $l$ states to fix an assignment to. So, for example, while $\operatorname{Pr}(G p)=0$,

\footnotetext{
${ }^{2}$ A language $L$ is a liveness language if $L=\Sigma^{*} \cdot L[1]$.
} 
we have that $\operatorname{Pr}_{1}(G p)=\frac{1}{2}, \operatorname{Pr}_{2}(G p)=\frac{1}{4}$, and in general $\operatorname{Pr}_{j}(G p)=\frac{1}{2^{j}}$. Indeed, an $l$-cycle satisfies $G p$ iff all its states satisfy $p$.

There are several interesting issues in the finite-state approach. First, it may seem obvious that the bigger $l$ is, the closer $\operatorname{Pr}_{l}(\varphi)$ gets to $\operatorname{Pr}(\varphi)$. This is, however, not so simple. For example, issues like cycles in $\varphi$ can cause $\operatorname{Pr}_{l}(\varphi)$ to be non-monotonic. For example, when $\varphi$ requires $p$ to hold in exactly all even positions, then $\operatorname{Pr}_{1}(\varphi)=$ $0, \operatorname{Pr}_{2}(\varphi)=\frac{1}{4}, \operatorname{Pr}_{3}(\varphi)=0, \operatorname{Pr}_{4}(\varphi)=\frac{1}{16}$, and so on.

Assume now that we have cleaned the cycle-based issue (for example by restricting attention to formulas without $X \mathrm{~s}$, or by restricting attention to cycles of "the right" length). Can we characterize safety properties by means of the asymptotic behavior of $\operatorname{Pr}_{l}(\varphi)$ ? Can we define different levels of safety according to the rate the probability decreases or increases? For example, clearly $\operatorname{Pr}_{l}(G p)$ tends to 0 as $l$ increases, whereas $\operatorname{Pr}_{l}(F p)$ tends to 1. Also, now, for a given $l$, we have that $\operatorname{Pr}_{l}(p W q)>\operatorname{Pr}_{l}(p U q)$. In addition, for a clopen property $\varphi$, we have that $\operatorname{Pr}_{l}(\varphi)$ stablizes once $l$ is bigger than the bound of $\varphi$. Still, the picture is not clean. For example, $F G p$ is a liveness formula, but $\operatorname{Pr}_{l}(F G p)$ decreases as $l$ increases. Finding a characterization of properties that is based on the analysis of $P r_{l}$ is an interesting question, and our initial research suggests a connection between the level of safety of $\varphi$ and the behavior of $\operatorname{Pr}_{l}(\varphi)$.

\section{References}

1. Alpern, B., Schneider, F.B.: Defining liveness. IPL 21, 181-185 (1985)

2. Alpern, B., Schneider, F.B.: Recognizing safety and liveness. Distributed Computing 2, $117-126$ (1987)

3. Barringer, H., Goldberg, A., Havelund, K., Sen, K.: Rule-based runtime verification. In: Steffen, B., Levi, G. (eds.) VMCAI 2004. LNCS, vol. 2937, pp. 44-57. Springer, Heidelberg (2004)

4. Beer, I., Ben-David, S., Eisner, C., Rodeh, Y.: Efficient detection of vacuity in ACTL formulas. In: Grumberg, O. (ed.) CAV 1997. LNCS, vol. 1254, pp. 279-290. Springer, Heidelberg (1997)

5. Biere, A., Cimatti, A., Clarke, E., Zhu, Y.: Symbolic model checking without BDDs. In: Cleaveland, W.R. (ed.) TACAS 1999. LNCS, vol. 1579, pp. 193-207. Springer, Heidelberg (1999)

6. Bloem, R., Gabow, H.N., Somenzi, F.: An algorithm for strongly connected component analysis in $n \log n$ symbolic steps. In: Johnson, S.D., Hunt Jr., W.A. (eds.) FMCAD 2000. LNCS, vol. 1954, pp. 37-54. Springer, Heidelberg (2000)

7. Courcoubetis, C., Vardi, M.Y., Wolper, P., Yannakakis, M.: Memory efficient algorithms for the verification of temporal properties. FMSD 1, 275-288 (1992)

8. Courcoubetis, C., Yannakakis, M.: The complexity of probabilistic verification. J. ACM 42, 857-907 (1995)

9. d'Amorim, M., Roşu, G.: Efficient monitoring of omega-languages. In: Etessami, K., Rajamani, S.K. (eds.) CAV 2005. LNCS, vol. 3576, pp. 364-378. Springer, Heidelberg (2005)

10. Ben-David, S., Kupferman, O.: A framework for ranking vacuity results. In: Van Hung, D., Ogawa, M. (eds.) ATVA 2013. LNCS, vol. 8172, pp. 148-162. Springer, Heidelberg (2013)

11. Ehlers, R., Finkbeiner, B.: Reactive safety. In: Proc. 2nd GANDALF. Electronic Proceedings in TCS, vol. 54, pp. 178-191 (2011)

12. Emerson, E.A.: Alternative semantics for temporal logics. TCS 26, 121-130 (1983)

13. Fagin, R.: Probabilities in finite models. Journal of Symb. Logic 41(1), 50-58 (1976) 
14. Filiot, E., Jin, N., Raskin, J.-F.: An antichain algorithm for LTL realizability. In: Bouajjani, A., Maler, O. (eds.) CAV 2009. LNCS, vol. 5643, pp. 263-277. Springer, Heidelberg (2009)

15. Gale, D., Stewart, F.M.: Infinite games of perfect information. Ann. Math. Studies 28, 245-266 (1953)

16. Greimel, K., Bloem, R., Jobstmann, B., Vardi, M.: Open implication. In: Aceto, L., Damgård, I., Goldberg, L.A., Halldórsson, M.M., Ingólfsdóttir, A., Walukiewicz, I. (eds.) ICALP 2008, Part II. LNCS, vol. 5126, pp. 361-372. Springer, Heidelberg (2008)

17. Gumm, H.P.: Another glance at the Alpern-Schneider characterization of safety and liveness in concurrent executions. IPL 47, 291-294 (1993)

18. Harel, D., Katz, G., Marron, A., Weiss, G.: Non-intrusive repair of reactive programs. In: ICECCS, pp. 3-12 (2012)

19. Harel, D., Pnueli, A.: On the development of reactive systems. In: Logics and Models of Concurrent Systems, NATO ASI, vol. F-13, pp. 477-498. Springer (1985)

20. Havelund, K., Roşu, G.: Synthesizing monitors for safety properties. In: Katoen, J.-P., Stevens, P. (eds.) TACAS 2002. LNCS, vol. 2280, pp. 342-356. Springer, Heidelberg (2002)

21. Kupferman, O., Lustig, Y., Vardi, M.Y.: On locally checkable properties. In: Hermann, M., Voronkov, A. (eds.) LPAR 2006. LNCS (LNAI), vol. 4246, pp. 302-316. Springer, Heidelberg (2006)

22. Kupferman, O., Vardi, M.Y.: Model checking of safety properties. In: Halbwachs, N., Peled, D.A. (eds.) CAV 1999. LNCS, vol. 1633, pp. 172-183. Springer, Heidelberg (1999)

23. Kupferman, O., Vardi, M.Y.: Model checking of safety properties. FMSD 19(3), 291-314 (2001)

24. Kupferman, O., Vardi, M.Y.: On bounded specifications. In: Nieuwenhuis, R., Voronkov, A. (eds.) LPAR 2001. LNCS (LNAI), vol. 2250, pp. 24-38. Springer, Heidelberg (2001)

25. Kupferman, O., Weiner, S.: Environment-friendly safety. In: Biere, A., Nahir, A., Vos, T. (eds.) HVC 2012. LNCS, vol. 7857, pp. 227-242. Springer, Heidelberg (2013)

26. Lamport, L.: Logical foundation. In: Alford, M.W., Hommel, G., Schneider, F.B., Ansart, J.P., Lamport, L., Mullery, G.P., Zhou, T.H. (eds.) Distributed Systems. LNCS, vol. 190, pp. 19-30. Springer, Heidelberg (1985)

27. Manna, Z., Pnueli, A.: he anchored version of the temporal framework. In: de Bakker, J.W., de Roever, W.-P., Rozenberg, G. (eds.) Linear Time, Branching Time and Partial Order in Logics and Models for Concurrency. LNCS, vol. 354, pp. 201-284. Springer, Heidelberg (1989)

28. Manna, Z., Pnueli, A.: The Temporal Logic of Reactive and Concurrent Systems: Specification. Springer (1992)

29. Manna, Z., Pnueli, A.: The Temporal Logic of Reactive and Concurrent Systems: Safety. Springer (1995)

30. Pnueli, A.: The temporal semantics of concurrent programs. TCS 13, 45-60 (1981)

31. Pnueli, A., Rosner, R.: On the synthesis of a reactive module. In: Proc. 16th POPL, pp. 179190 (1989)

32. Pnueli, A., Shahar, E.: Liveness and acceleration in parameterized verification. In: Emerson, E.A., Sistla, A.P. (eds.) CAV 2000. LNCS, vol. 1855, pp. 328-343. Springer, Heidelberg (2000)

33. Sistla, A.P.: Safety, liveness and fairness in temporal logic. Formal Aspects of Computing 6, 495-511 (1994)

34. Sistla, A.P., Clarke, E.M.: The complexity of propositional linear temporal logic. Journal of the ACM 32, 733-749 (1985)

35. Touati, H.J., Brayton, R.K., Kurshan, R.: Testing language containment for $\omega$-automata using BDD's. I \& C 118(1), 101-109 (1995)

36. Vardi, M.Y., Wolper, P.: Reasoning about infinite computations. I \& C 115(1), 1-37 (1994) 